\title{
Ultrahigh Brightness Bunches from Hybrid Plasma Accelerators as Drivers of 5th Generation Light Sources
}

B. Hidding ${ }^{1,2,3}$, G. Manahan ${ }^{1}$, O. Karger ${ }^{2}$, A. Knetsch ${ }^{2}$, G. Wittig ${ }^{2}$, D. Jaroszynski ${ }^{1}$, Z.-M. Sheng ${ }^{1}$, Y. $\mathrm{Xi}^{3}{ }^{3}$, A. Deng ${ }^{3}$, J.B. Rosenzweig ${ }^{3}$, G. Andonian ${ }^{3,4}$, A. Murokh ${ }^{4}$, G. Pretzler ${ }^{5}$, D.L. Bruhwiler ${ }^{6,7}$, J. Smith $^{8}$

${ }^{1}$ Department of Physics, SUPA, Strathclyde University,

Glasgow, UK, G4 ONG, ${ }^{2}$ Department of Experimental Physics,

University of Hamburg \& $C F E L$, Germany, ${ }^{3}$ Department of Physics and Astronomy,

University of California, Los Angeles, USA, ${ }^{4}$ RadiaBeam Technologies,

Santa Monica, USA, ${ }^{5}$ Institute of Laser and Plasma Physics,

University of Düsseldorf, Germany, ${ }^{6}$ University of Colorado at Boulder,

Center for Integrated Plasma Studies, Boulder, Colorado 80309,

USA, ${ }^{7}$ RadiaSoft LLC, Boulder, Colorado 80304, USA,

8 Tech-X Ltd., Daresbury, Cheshire, WA4 4FS, UK

(Dated: September 17, 2014)

Spiking electron beam driven plasma waves with novel laser-driven underdense photocathodes can produce electron witness bunches with extreme brightness. The development of such hybrid systems and their potential as future plasma-based accelerators and compact yet high performance light sources is discussed. 


\section{INTRODUCTION}

Cathode ray tubes were essential in the discovery of x-rays [1] as well as in the discovery of plasma [2-4]. The electron beams originating from these cathode ray tubes - the first man-made electron accelerators - are naturally limited in quality and energy. Since then, the many advances in accelerator physics have continuously enabled more and more sophisticated generations of electron accelerators. This progress is characterized by a dramatic increase in the obtainable electron energy $E$ and brightness $B=2 I / \epsilon_{n}^{2}$, where $I$ is the electron beam current and $\epsilon_{n}$ the normalized emittance. New generations of electron accelerators often spawn a new generation of light sources. Using electron density oscillations in plasma - the very medium discovered by the first electron accelerators - to produce a new generation of electron "accelerators for very high current and super-high energies of the order of $10^{12} \mathrm{eV}$ and even more" [5], is today a vibrant research field that many believe to have the potential for a next-generation electron accelerator, which in turn may pave the way for a new generation of light sources.

Such plasma waves are characterized by the plasma frequency $\omega_{p}=\left[n_{e} e^{2} /\left(m_{e} \epsilon_{0}\right)\right]^{-1 / 2}$, where $n_{e}$ is the electron plasma density, $e$ is the electron charge, $m_{e}$ the electron mass and $\epsilon_{0}$ the vacuum permittivity. They can either be driven by intense laser pulses as in laser wakefield acceleration (LWFA) [6], or by intense particle bunches in plasma wakefield acceleration (PWFA) [7,8] scenarios. While there are many similarities in LWFA and PWFA, there are also profound differences. In the following, therefore electron beam driven plasma cavities are consequently and for historical reasons denoted blowout, whereas for laser-driven plasma cavities the term bubble [9] is used.

A main difference is, that laser pulses are electromagnetic waves with electric and magnetic fields oscillating in the transverse directions, whereas electron bunches have unidirectional electric fields, which is schemtically shown in figure 1. It is intuitively clear that unidirectional electric fields are much better suited to expel electrons off axis when compared to rapidly oscillating fields. This is because the ponderomotive force is a second-order effect $[10,11]$. At a laser wavelength of $\approx 1 \mu \mathrm{m}$, intensities in the range of $I \approx 10^{18}-10^{19} \mathrm{~W} / \mathrm{cm}^{2}$, corresponding to a normalised vector potential $a_{0}=\frac{e E}{m_{e} \omega c}>1$, are therefore required in order to expel the electrons via the ponderomotive force $F_{p} \propto a^{2}$ and to produce an electron-free plasma cavity with a single laser pulse. The peak electric fields connected to an $a_{0}=1$, which are today achievable with Ti:sapphire lasers of wavelength $\lambda=0.8 \mu \mathrm{m}$, amount to $E_{0}=a_{0} \frac{2 \pi m_{e} c^{2}}{e \lambda} \approx a_{0} / \lambda[\mu \mathrm{m}] \times 3.2 \times 10^{12}[\mathrm{~V} / \mathrm{m}] \approx 4 \mathrm{TV} / \mathrm{m}$. At these electric field values, even higher ionization states of matter are completely ionized. This can be approximated using the barrier suppression ionization intensity threshold $I_{\mathrm{BSI}} \approx 4 \times 10^{9} \frac{\xi_{i}^{4}[\mathrm{eV}]}{Z^{2}}$. Hydrogen, for example, has an ionization potential of $\xi_{i}=13.6 \mathrm{eV}$ and is completely ionized already at an intensity of $I \approx 1.4 \times 10^{14} \mathrm{~W} / \mathrm{cm}^{2}$. With typical pulse durations of the order of $\tau \approx 30 \mathrm{fs}$, a plasma wave is excited optimally for a laser pulse duration that matches the plasma wavelength $\lambda_{p} \approx 2 c \tau$ [12]. Laserplasma-accelerators typically operate at somewhat lower densities because the energy gain is limited due to dephasing, diffraction and injection; a typical value is a plasma electron density of $n_{e} \approx 4 \pi^{2} c^{2} \epsilon_{0} m_{e}\left(\lambda_{p}^{2} e^{2}\right)^{-1} \approx 10^{18} \mathrm{~cm}^{-3}$.
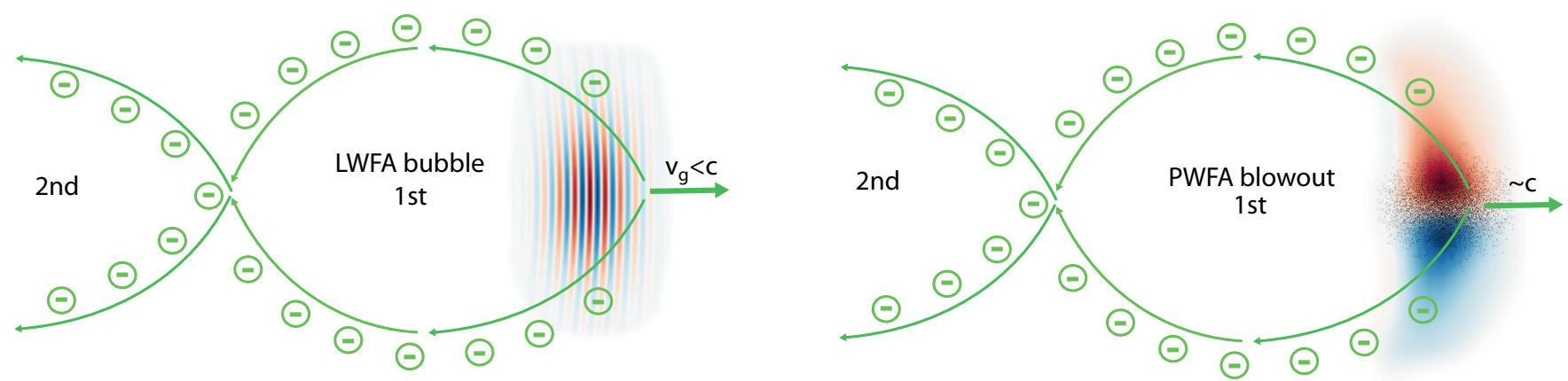

FIG. 1: Schematic view on plasma wave excitation driven by a laser pulse (left), exciting a bubble, and an electron pulse (right) exciting a blowout.

Electron bunches, on the other hand, can excite nonlinear plasma wave blowouts as long as the bunch electron density is larger than the plasma density $n_{b}>n_{e}$. For an axially symmetric Gaussian bunch profile with bunch length $\sigma_{z}$ and bunch radius $\sigma_{r}$, the bunch density

$$
n_{b}=\frac{Q}{(2 \pi)^{3 / 2} \sigma_{r}^{2} e \sigma_{z}}
$$

can satisfy $n_{b} \geq n_{e} \approx 10^{18} \mathrm{~cm}^{-3}$ for bunch parameters of charge $Q=N e=100 \mathrm{pC}, \sigma_{r} \approx 2 \mu \mathrm{m}$ and $\sigma_{z} \approx 4 \mu \mathrm{m}$. The 
peak electric field of such a bunch can be approximated as

$$
E_{r}\left(r=\sigma_{r}\right) \approx \frac{Q}{\sigma_{z} \epsilon_{0} \sigma_{r}} \times 0.025
$$

For the above parameters, these fields amount to $E_{r} \approx 35 \mathrm{GV} / \mathrm{m}$ - which is much less than the ionization threshold of helium, for example. A general conclusion is that ionization is much easier with laser pulses than with electron pulses.

As regards longitudinal driver bunch duration, there are similarities between methods of compression of high-power laser pulses and the compression of an electron bunch. With laser pulses, one introduces a correlated longitudinal energy chirp to the broadband laser pulse in a stretcher prior to amplification to prevent destruction of the amplifier medium. After amplification, the stretched pulse is re-compressed in a dispersive system, for example in a grating compressor. This chirped pulse amplification (CPA) [13] method is an enabling technology for the whole field of high intensity laser science. Electron bunches are generated in state-of-the-art photoguns with a short laser pulse (typically, a Ti:sapphire laser pulse at fundamental wavelengths or harmonics) incident on the photocathode material, where the photo-electrons are released. Radio-frequency fields are used to extract the electrons. Depending on their individual phase in the accelerating radio-frequency wave, electrons may also develop a correlated energy spread. At low energies $\lesssim 5 \mathrm{MeV}$, the large differences in velocities $\beta$ (compare figure $2 \mathrm{~b}$ ) can be exploited for active or passive velocity bunching (e.g. in a drift tube) to compress the electron bunches longitudinally. This increases the peak current, and the charge density of the electron bunch, which is highly advantageous for driving a plasma wave.

The transverse expansion of electron bunches and laser pulses during propagation in vacuum follows a hyperbolic profile in both cases. The Gaussian laser beam propagating in the z-direction diffracts as

$$
w(z)=w_{0} \sqrt{1+\left(\frac{z}{Z_{R}}\right)^{2}}
$$

where

$$
Z_{R}=\pi w_{0}^{2} / \lambda
$$

is the Rayleigh length, which depends on the focus radius $w_{0}$ and the laser wavelength $\lambda$. High-power laser pulses with intensities in the $I \approx 10^{18}-10^{19} \mathrm{~W} / \mathrm{cm}^{2}$ range required for laser wakefield acceleration (LWFA), are almost exclusively based based on Ti:sapphire, which fixes the central wavelength to $\lambda \approx 800 \mathrm{~nm}$. Today's typical laser power levels of a few hundreds of TW require spot sizes $w_{0}$ of a few microns to reach intensities of $a_{0}>1$. Assuming a spot size of $w_{0}=10 \mu \mathrm{m}$, for example, this leads to Rayleigh lengths of $Z_{R} \approx 400 \mu \mathrm{m}$. This is the characteristic propagation length scale of the laser beam in vacuum. A diffracting laser pulse decreases the intensity, which limits the acceleration length of LWFA. Fortunately, relativistic self-focussing in plasma can guide the laser pulse over many Rayleigh lengths, and active guiding in plasma channels can counteract the diffraction process, allowing cm acceleration distances and GeV energies to be reached with state-of-the-art laser systems [14-16].

Electron bunch diameters evolve as

$$
\sigma_{r}(z)=\sigma_{r 0} \sqrt{1+\left(\frac{z}{\beta^{*}}\right)^{2}}
$$

where the beta function length

$$
\beta^{*}=\sigma_{r 0}^{2} \gamma / \epsilon_{n}
$$

is dependent on electron bunch diameter at focus $\sigma_{r 0}$, its Lorentz factor $\gamma=\left(1-v^{2} / c^{2}\right)^{-1 / 2}$, and its normalized emittance $\epsilon_{n}$. Assuming a focus diameter of $\sigma_{r 0}=10 \mu \mathrm{m}$ as in the laser pulse example, an electron bunch energy of $E=m_{0} c^{2}(\gamma-1)=1 \mathrm{GeV}$ and a typical normalized emittance of $\epsilon_{n}=10^{-6} \mathrm{mrad}$, leads to a beta function length scale of $\beta^{*} \approx 20 \mathrm{~cm}$. This is many orders of magnitude longer than in the laser pulse case, where the laser wavelength $\lambda$ is fixed and only the spot size $w_{0}$ is a variable. In contrast, with electron bunches, in addition to the spot size, the electron bunch kinetic energy $E$ and the emittance $\epsilon_{n}$ are variables and can be optimized to obtain even longer beta function lengths. In effect, this means that proper electron bunches can drive a plasma wave over much longer distances than laser pulses, allowing for enhanced acceleration lengths. Indeed, energy doubling of $42 \mathrm{GeV}$ electrons has been demonstrated at SLAC in an electron-beam driven PWFA setup on metre-scale length [17]. Figure 2 a) compares the transverse diffraction of typical laser pulses and electron pulses in vacuum, which visualizes the much 
longer relevant acceleration lengths obtainable with electron pulses, and in turn the ability of laser pulses to ionize highly locally confined volumes.

Finally, relativistic electron bunches propagate with practically the same velocity in vacuum as in plasma, which is $\approx c$ for sufficiently relativistic electron bunches. In contrast, laser pulses of frequency $\omega_{0}$ propagate at their group velocity

$$
v_{g}=c\left(1-\frac{\omega_{p}^{2}}{\omega_{0}^{2}}\right)^{1 / 2}
$$

which is significantly slower than the speed of light in vacuum $c$ and of the propagation speed of relativistic electrons in vacuum or plasma $\approx c$. This leads to substantial dephasing effects encountered in LWFA, whereas there is no significant dephasing in PWFA, where both driver and witness electrons propagate with approximately the same speed $c$. This is shown in figure $2 \mathrm{~b}$ ).

a)

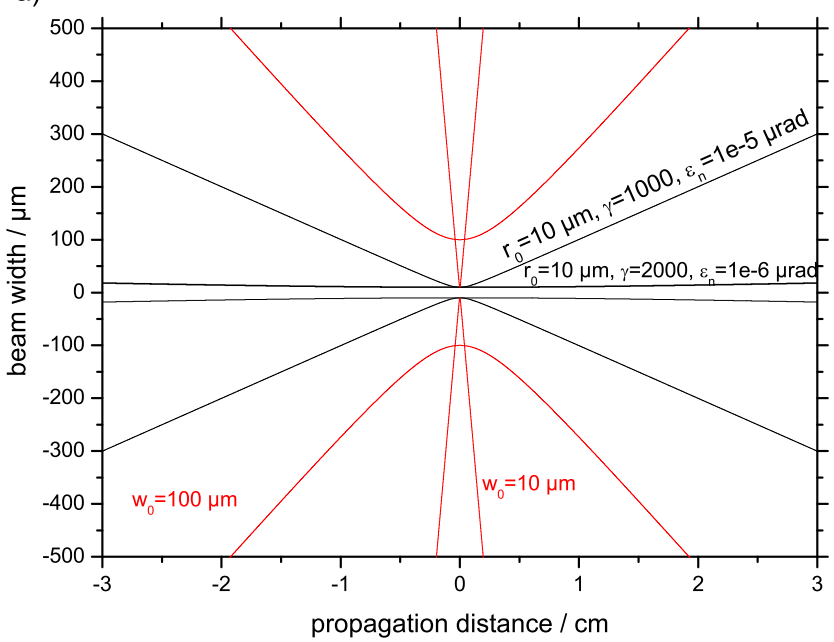

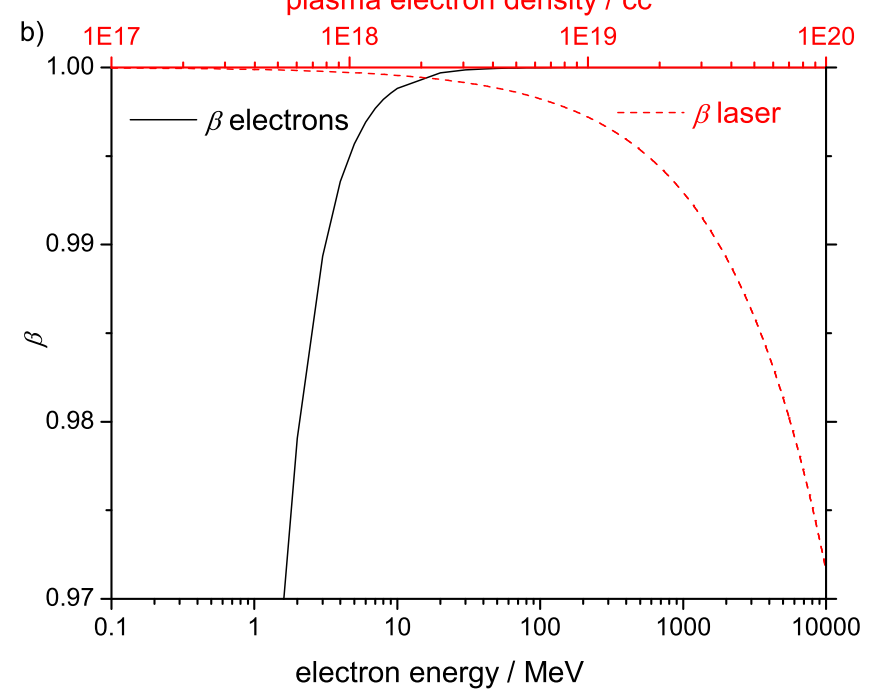

FIG. 2: Transverse beam expansion (a) and longitudinal propagation velocity (b) of Gaussian laser pulses and electron beams in vacuum. a) Electron pulses (black lines) of relativistic energies and typical emittance (e.g. $\gamma=2000, \epsilon_{n}=10^{-6} \mathrm{mrad}$, but even for $\gamma=1000, \epsilon_{n}=10^{-5} \mathrm{mrad}$ ), and expand much slower than laser pulses (red) with $w_{0}=10 \mu \mathrm{m}$ or even $w_{0}=100 \mu \mathrm{m}$. b) The propagation velocities of electron bunches in plasma are substantially

faster than for laser pulses, especially at elevated plasma electron densities. Space charge, plasma lensing and focusing effects are not included.

Along general lines, these differences between laser pulses and electron bunches indicate that suitable bunches can inherently provide a much longer usable acceleration length than laser pulses. While more subtle effects and techniques such as space charge (an effect which importance decreases $\propto \gamma^{2}$ ), head erosion, self-focusing and plasma lensing, longitudinal and transverse plasma tapering are not regarded here, these broad strokes indicate that electron bunch drivers are much better as drivers of plasma waves. On the other hand, laser pulses are much better at (selectively) ionizing media.

\section{TROJAN HORSE UNDERDENSE PHOTOCATHODE PWFA}

From a general comparison and analysis of advantages and disadvantages of electron and laser pulses for plasma wakefield acceleration, we developed a stratagem designed to exploit the best of both worlds, LWFA and PWFA. Fortunately, the advantages and disadvantages of electron and laser pulses are almost completely complementary. This led to a hybrid scheme patented in 2011 [18], published in 2012 [19] and eventually popularized as the "Trojan Horse" underdense photocathode concept [20]. The core idea is to use electron bunches as drivers to set up the plasma blowout cavity, exploiting the practically dephasing-free, long acceleration lengths, and to use laser pulses for locally confined release of ultracold electrons directly within the blowout. These high-quality witness electrons 
are then accelerated in the giant electric plasma fields. The electron bunch driver sets up a plasma wave based on a low ionization threshold media component (LIT) such as hydrogen, which is either pre-ionized, or self-ionized by the electric fields of the electron bunch driver. As seen above, the electric fields of typical electron bunch drivers are hardly sufficient to ionize even hydrogen with its low ionization potential of $13.6 \mathrm{eV}$, which leaves components with a higher ionization threshold (HIT) such as helium $(24.6 \mathrm{eV})$ uncompromised. In a mixture of hydrogen and helium, for example, the hydrogen will be fully ionized, and the bunch driver will excite a plasma wave based on hydrogen electron displacement from the hydrogen ions, while neutral helium gas will remain uniformly present in the mixture, according to the gas laws. This HIT reservoir is in the next step harvested by focusing a synchronized laser pulse with short (effective) Rayleigh length into the inside of the LIT plasma blowout to a peak intensity just sufficient to liberate HIT electrons via ionization. For helium, for example, Ti:sapphire laser pulse intensities of $I_{\mathrm{BSI}} \approx 10^{15} \mathrm{~W} / \mathrm{cm}^{2}$ are sufficient (note that the BSI threshold is an upper limit and that ADK ionization is active at considerable lower intensities [20]. Obtaining such sub-relativistic focus intensities are easily achieveable with laser energies $<100 \mu \mathrm{J}$ at pulse durations of a few or a few tens of femtoseconds, when strongly focused to spot sizes of $w_{0}$ of a few $\mu$ m. By tuning the effective focal volume and the laser intensity, electrons are released in an arbitrarily small volume. In diametral contrast to LWFA, strong focusing and a thus strongly limited interaction length is highly favourable (ionization defocusing effects may help in this regard). Because the laser pulse intensity is at the same time orders of magnitude lower than the laser pulse intensities in LWFA, the phase space volume taken by the released electrons is also orders of magnitude smaller. This is the key to producing ultralow emittance, high brightness electron bunches.

$$
\text { a) }
$$

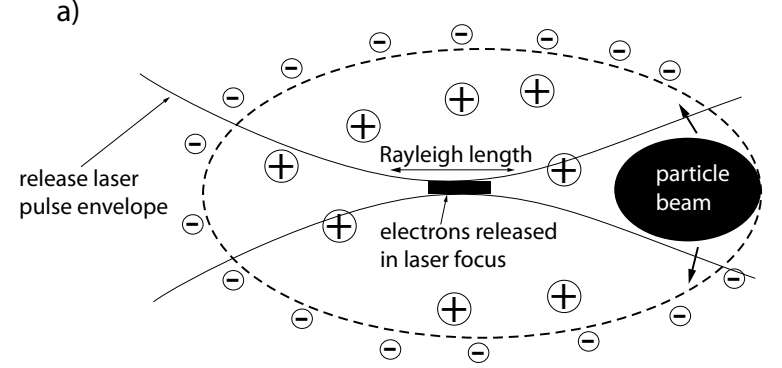

c)

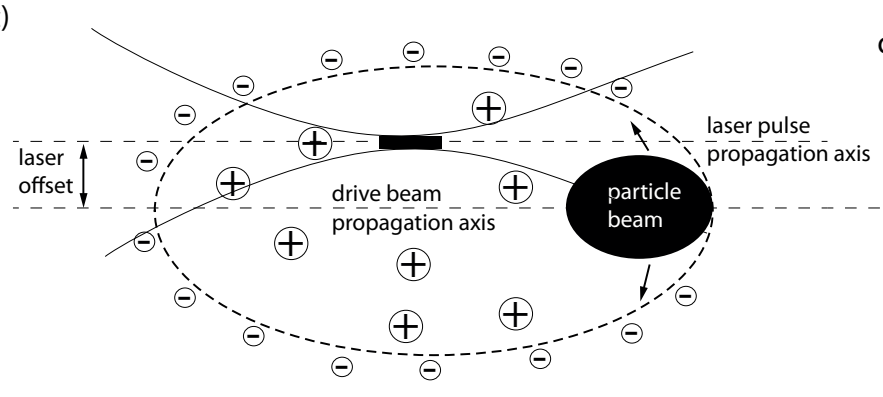

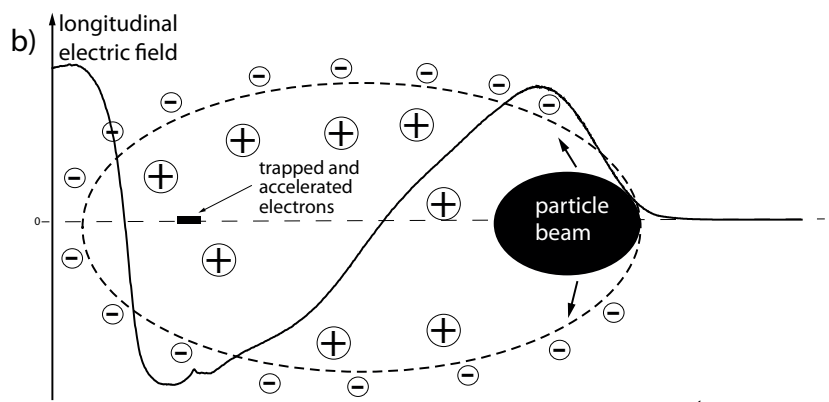

d)

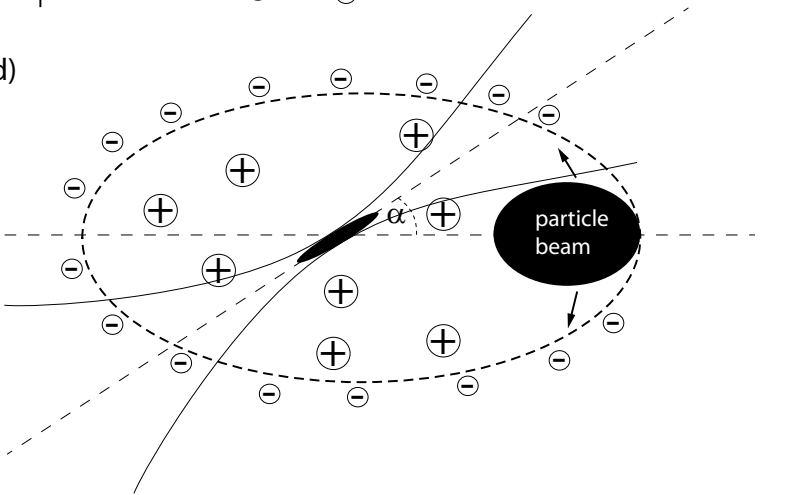

FIG. 3: Schematic underdense photocathode process (from [18]). a) HIT electron release by laser pulse, b) trapped HIT electron bunch, c) offset injection and d) injection at an arbitrary angle.

Figure 3 visualizes how the process was envisioned by using figures from the original patent work [18]. The particle beam driver (in most cases an electron bunch, but it could in principle also be a proton bunch [21, 22]) travels to the right and sets up a plasma wave in its wake, based on the LIT medium. The synchronized laser pulse, strongly focused to an intensity matching the ionization threshold of the HIT component, releases HIT electrons (indicated by the black rectangle in the picture) in the laser focus at arbitrary position in the blowout (figure 3 a). These electrons, subject to the plasma blowout's trapping potential, are at first left behind as the blowout moves forward in the wake of the bunch driver, but as they experience the accelerating longitudinal field (see lineout in figure $3 \mathrm{~b}$ ) they are accelerated and eventually trapped, if the trapping potential is sufficient. From a practical point of view, this is already the case when the electron reach $\mathrm{MeV}$ energies even when the driver beam may have $\mathrm{GeV}$ energies, because of the insignificant differences in $\beta$ (compare figure $2 \mathrm{~b}$ ) along with the comparably short acceleration distances. At the same time, the electrons experience the plasma-based analogue of active velocity bunching, as the longitudinal electric field in the plasma blowout has a positive gradient $d E / d x$ in the longitudinal direction [23]. Therefore, during 
the trapping process, the electrons are bunched and compressed in the longitudinal (and transverse) direction, which is reflected by the smaller rectangle representing the compressed, trapped electrons in figure $3 \mathrm{~b}$ ). Depending on the total charge that is released by the laser pulse, the electric field inside the plasma blowout may be distorted due to beam loading effects $[24,25]$. This can flatten the effective accelerating electric field locally (see figure 3 b) and is a strategy towards reduction of energy spread of the witness bunch. While figure 3 a) and b) depict the release of electrons on axis collinear with the drive beam, it is also possible to release off axis figure 3 c), which will lead to forced betatron oscillations of the electrons around axis [19] and/or with the laser pulse at an arbitrary angle (figure $3 \mathrm{~d}$ ), which then will have the laser pulse duration effect the produced electron bunch duration.

The electron-liberating Trojan laser pulse has direct effect on the electron bunch, which allows advanced electron bunch synthesis and shaping. As regards flexibility and tunability, this process may be even superior to the stateof-the-art solid photocathodes that are used in modern rf cavity based accelerators. One main difference is that in a conventional photocathode the laser pulse is absorbed and reflected because the photocathode material is overdense with solid electron density orders of magnitude above the critical density $n_{c}(\omega)=\epsilon_{0} m_{0} \omega^{2} / e^{2} \approx 1.76 \times 10^{21} \mathrm{~cm}^{-3}$ for a Ti:sapphire laser wavelength or $n_{c}(3 \omega) \approx 2.1 \times 10^{22} \mathrm{~cm}^{-3}$ at the third harmonic of $\lambda \approx 266 \mathrm{~nm}$.

In case of the Trojan Horse method, the medium is underdense to such laser pulses, allowing them to propagate through the photocathode material as long as the ionization threshold is not reached. In principle, the HIT component therefore can be present in a concentration of up to the critical density $n_{c}$, which then produces extremely high charge density per unit volume. Since, at such densities, the corresponding plasma wavelength $\lambda_{p}$ is much less than 1 micron, such extreme densities (which would likely lead to laser beam filamentation as soon as ionization sets in) would require the HIT density concentration to be independent of the LIT density. This is the case in case for two or multiple gas components, for example hydrogen (LIT) and helium (HIT). In contrast, when a higher ionization level of the LIT species is used as HIT level, such as with the first and second ionization thresholds of an alkali metal such as $\mathrm{Rb}$, or when using $\mathrm{He}^{+}$and $\mathrm{He}^{++}$, restrictions would arise because the required LIT plasma wavelength. From this viewpoint, the use of $\mathrm{H}$ and $\mathrm{He}$ is attractive because their concentrations are independently tunable [19]. At constant hydrogen gas density, for example, increasing the helium density will lead to an increased electron witness bunch charge. Next to the HIT medium density, tuning the laser pulse intensity, focusing, frequency, polarization etc. are other parameters that can be used to steer the witness bunch production meticulously, thus justifying the term "underdense photocathode". Many of these parameters have a direct analogy with solid photocathode guns. However, the spatial scales are very different, corresponding to the difference in the size of metallic cavities compared with the plasma blowout cavity. For example, the laser spot size on a conventional photocathode is typically a few mm squared, while laser spot sizes in underdense photocathodes are generally as small as is possible. In underdense photocathodes, the use of higher frequencies allows for smaller spot sizes $w_{0} \propto \lambda$, which also reduces the Rayleigh length $Z_{R} \propto w_{0}^{2} / \lambda$. At the same time, the electric field that is needed for ionization, which is purely material dependent, scales as $E_{0} \propto a_{0} / \lambda$. Therefore at shorter wavelengths, the required threshold intensity $a_{0} \propto \lambda$. The initial phase space volume of the released electron bunch depends on the transverse diameter of the electron bunch and their transverse momenta. Both can be reduced by using higher laser frequencies in the underdense photocathode $[20,23]$. The transverse initial diameter $\sigma_{r}$ is approximately the laser pulse spot size $w_{0} / \sqrt{2}$, and the transverse momentum $p_{y, z} /(m c) \approx a_{0} / 2$. The obtainable transverse emittance $\epsilon_{n} \approx \sigma_{r} p_{y, z} /(m c) \approx w_{0} a_{0} / 2^{3 / 2}[19]$ is therefore proportional to both the spot size $w_{0}$ and the vector potential $a_{0}$. The residual laser contribution to the transverse normalized emittance can therefore be decreased using higher laser frequencies. If the beam parameter product can be conserved during frequency up-conversion of ultra-short laser pulses, not only the spot size, but also the Rayleigh length decreases at shorter laser wavelengths. In turn, this also decreases the emittance growth due to phase mixing processes [23, 26], which occurs during the bunch generation, bunching and trapping due to different longitudinal birth positions of electrons in the underdense photocathode.

3D simulations have been carried out using the particle-in-cell code VSim/VORPAL [27] for an electron beam driver bunch from a conventional accelerator with a total charge of $Q=1 \mathrm{nC}$, a duration of $\sigma_{z}=30 \mu \mathrm{m}$, corresponding to an r.m.s. current of $I \approx 7 \mathrm{kA}$ and a width of $\sigma_{r}=20 \mu \mathrm{m}$, the electron energy is $23 \mathrm{GeV}$ and the emittance is $\epsilon_{n}=2.25 \times 10^{-6} \mathrm{mrad}$, consistent with the above examples and (despite the lower charge) with the typical performance of the FACET electron bunch at SLAC. The underdense medium consists of a mixture of hydrogen and helium, which although independently tunable are here both set to $n_{e}=5 \times 10^{16} \mathrm{~cm}^{-3}$ for simplicity. The hydrogen is pre-ionized, and the electron bunch driver sets up a LIT plasma wave with a plasma wavelength of $\lambda_{p} \approx 150 \mu \mathrm{m}$. The Trojan photocathode laser pulse is Gaussian at the standard Ti:sapphire wavelength of $\lambda=0.8 \mu \mathrm{m}$ with a spot size of $w_{0}=4 \mu \mathrm{m}$, corresponding to a Rayleigh length of $Z_{R} \approx 60 \mu \mathrm{m}$, and the pulse duration is $\tau=40$ fs. The laser pulse is represented by a mathematical function: a) transversely, a Gaussian envelope with curved wavefronts that satisfy the paraxial approximation; b) longitudinally, convolution with another Gaussian envelope that varies on a spatial scale long compared to the wavelength; and c) temporally, propagation at the vacuum group velocity, with focusing and diffraction governed by the paraxial approximation, and with zero longitudinal dispersion. Because the normalized laser pulse amplitude is much less than unity, the plasma response will be that of a linear dielectric, as 
0

\section{a)}

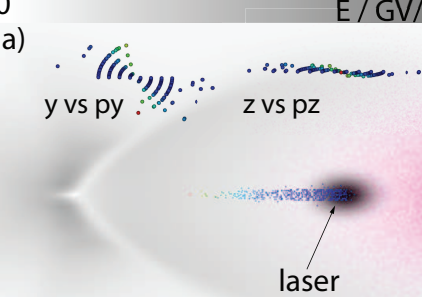

\section{b)}

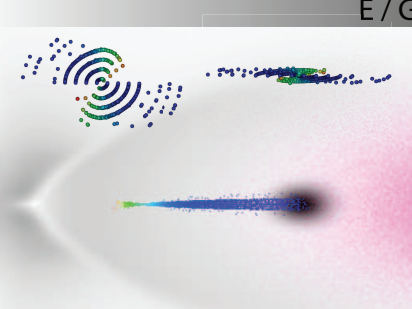

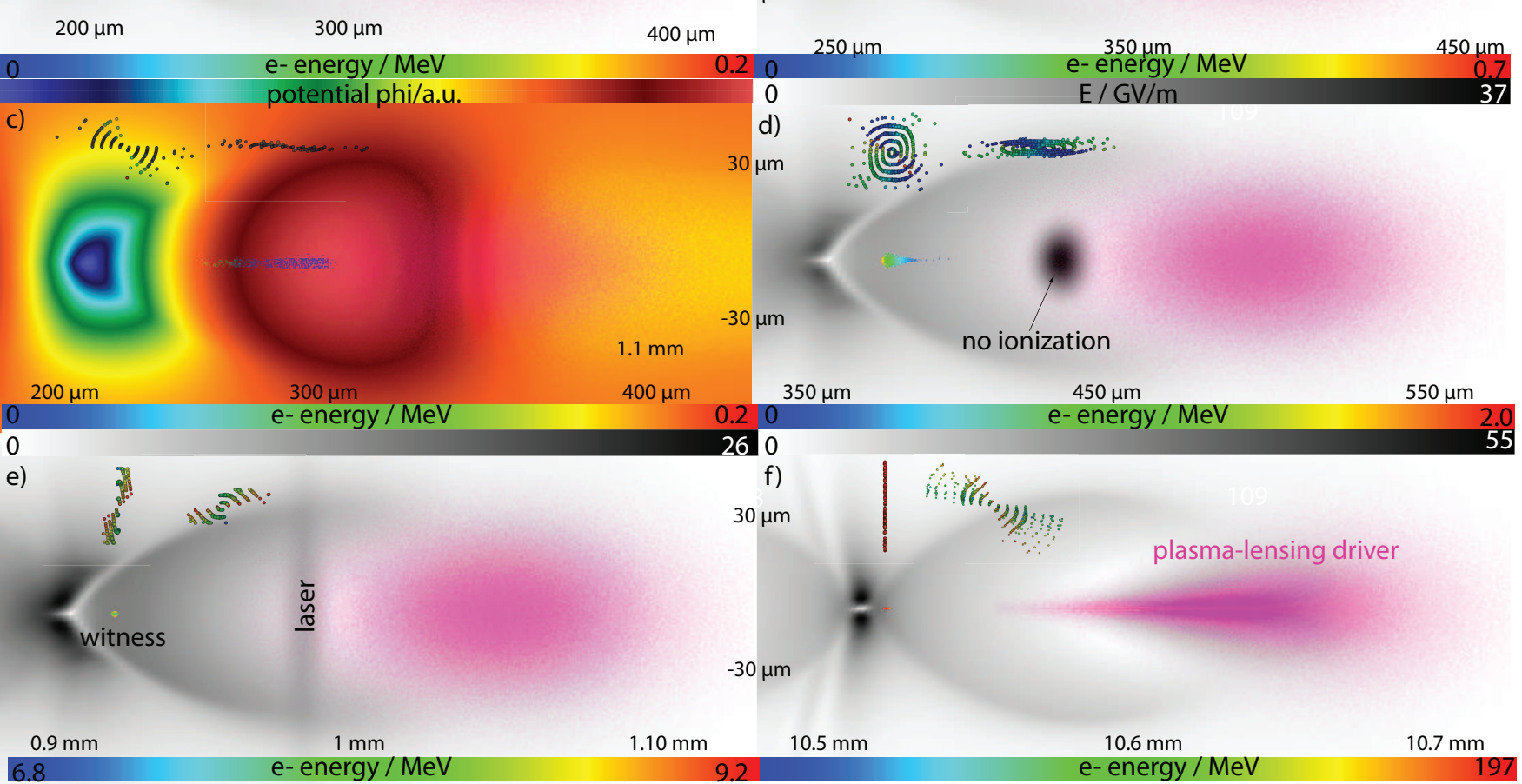

FIG. 4: 3D PIC simulation results with VORPAL/VSim. The Gaussian drive bunch (pink) moves to the right and sets up the H plasma wave. He is ionized by the strongly focused laser pulse at the wave's trapping potential maximum, releasing electrons in figures a)-c) until it is diffracted to below the He ionization threshold. Then the witness bunch is fully formed and compresses longitudinally (d) and is then further accelerated (e-f). The insets show the He electrons' transverse phase space distribution $y$ vs. $p_{y}$ and $z$ vs. $p_{z}$, respectively.

long as the plasma frequency remains small compared to the laser frequency along the path of the laser pulse, which can be largely satisfied by injecting slightly off-axis with respect to the electron beam propagation. Hence, the explicit approximations regarding group velocity and use of the paraxial approximation in vacuum are reasonable and will be modest compared to experimental timing and steering jitter. The laser intensity is varied from $a_{0}=0.022$ to $a_{0}=0.016$ for the same focusing, which in practice can be easily done by using an energy degrader, for example. 3D PIC simulations that fully resolve the laser wavelength are computationally very expensive. Versatile simulation input decks have been developed where the laser pulse can either be fully resolved, approximated by an envelope or by the paraxial approximation, the frequency and polarization can be tuned, and the electron bunch driver can be approximated either by static, analytic current distribution or by variable or constant weight macro-particle distribution. Here, the laser pulse is approximated by its envelope, which allows for a simulation speed-up of many orders of magnitude. While the ionization physics are compatible with this approach, the residual canonical momentum imposed on the released electrons is not accurately modelled. As a result, the observed emittance underestimates the real obtainable emittance, but on the other hand allows to explore the effects of space charge and phase mixing independently of the laser kick. Figure 4 shows results of a simulation for a laser intensity of $a_{0}=0.022$, a value approaching the BSI ionization intensity for helium of $a_{0}=0.026$. The laser pulse is in collinear geometry and follows the electron driver bunch (pink) on axis in a distance of $\approx 50 \mu \mathrm{m}$. Shown in figure $4 \mathrm{a}$ ) is the electric field magnitude 
in the simulation box, which allows to observe the transversely polarized focusing laser pulse as well as the hydrogen plasma blowout shape and its length of approximately $\lambda_{p} \approx 150 \mu \mathrm{m}$. In the first snapshot 4 a), the laser pulse has just reached its focus, producing a peak electric field of $E \approx 81 \mathrm{GV} / \mathrm{m}$ and has started releasing He electrons, which are falling behind. The corresponding electric potential is displayed in $4 \mathrm{c}$ ), where one can see that the electron release happens approximately at the electrostatic wave's potential minimum, which is optimal for trapping. The insets in the top left corner of each snapshot show the transverse phase space distribution of the generated witness bunch particles. In the next snapshot $4 \mathrm{~b}$ ), the plasma wave and the laser pulse have progressed approximately $50 \mu \mathrm{m}$, and the laser pulse continues to ionize helium at an electric field of $E \approx 81 \mathrm{GV} / \mathrm{m}$, releasing further charge from the HIT helium gas component. The first electrons are already trapped and accelerated to maximum energies of $0.7 \mathrm{MeV}$. After propagating another $80 \mu \mathrm{m}$ (see figure $4 \mathrm{~d}$ ), the laser pulse has diffracted and has lost its HIT ionization capabilities, in this snapshot already being decreased to a peak electric field of $E \approx 37 \mathrm{GV} / \mathrm{m}$. The released He electrons have a total charge of $Q \approx 110 \mathrm{pC}$, are almost all bunched, and peak energies reach $2.0 \mathrm{MeV}$. After nearly one mm of propagation (figure $4 \mathrm{e}$ ), the witness bunch is compressed to a very small longitudinal and transverse size, the laser has diffracted further, and the peak electron energy has reached $9 \mathrm{MeV}$. Next, the situation is depicted after $\approx 10$ $\mathrm{mm}$ of total propagation, where the peak electron witness bunch energy is $\approx 200 \mathrm{MeV}$, and the latter part of the electron bunch driver shows substantial self-lensing due to the transverse forces exerted by the plasma blowout.

The driver bunch has not only transferred energy to the witness bunch via the plasma, but simultaneously a "brightness transformation" takes place. This is visualized in figure 5, where in a) the evolution of the witness bunch normalized emittance and the corresponding brightness is plotted. During the whole process, the witness bunch does not exceed an emittance observed in the simulation of $\epsilon_{n} \approx 1.5 \times 10^{-8} \mathrm{mrad}$, and after the bunch underdense photocathode process is completed and the bunch is fully captured and compressed, the observed emittance reduces down to $\epsilon_{n} \approx 5 \times 10^{-9} \mathrm{mrad}$. However, the laser envelope approximation does not properly take into account the laser kick contribution to the emittance, which according to the crude scaling is $\epsilon_{n} \propto w_{0} a_{0}$, which predicts $\epsilon_{n} \approx \sigma_{r} p_{y, z} /(m c) \approx w_{0} a_{0} / 2^{3 / 2} \approx 3 \times 10^{-8} \mathrm{mrad}$, which is an order of magnitude larger and suggests that the laser kick contribution to the emittance strongly dominates here over other effects such as space charge even at the rather large witness bunch charge of $100 \mathrm{pC}$. In figure $5 \mathrm{a}$ ), the emittance, as observed in the simulation, is produced by adding the value from the analytical estimate $\epsilon_{n} \approx 3 \times 10^{-8} \mathrm{mrad}$ to the emittance observed in the simulation. Now, this corrected emittance value is overestimated. For example, here it is assumed that the laser kick is in both transverse directions, while in case of linear polarization the laser kick will initially only increase the emittance in one direction. Therefore the laser kick contribution would be decreased substantially in reality. Here, we neglect these effects for the sake of a conservative estimate in the context of the reduced physics model used in the 3D simulations. Nevertheless, the corresponding brightness values are impressive: the peak brightness reaches $B=2 I / \epsilon_{n}^{2} \approx 1.2 \times 10^{20} \mathrm{Am}^{-2} \mathrm{rad}^{-2}$. This is partially because of the huge witness currents that propagate in the plasma, which amount to $I \approx 70 \mathrm{kA}$. Figure $5 \mathrm{~b}$ ) shows the driver bunch emittance decay, which progresses almost linearly, from the initial value of $\epsilon_{n} \approx 2.25 \times 10^{-6} \mathrm{mrad}$ and increasing to $\epsilon_{n} \approx 2.5 \times 10^{-4} \mathrm{mrad}$ over a distance of $6 \mathrm{~mm}$. The brightness, accordingly, decreases by four orders of magnitude from $B \approx 2 \times 10^{15} \mathrm{Am}^{-2} \mathrm{rad}^{-2}$ to $B \approx 2 \times 10^{11} \mathrm{Am}^{-2} \mathrm{rad}^{-2}$. The witness bunch brightness is therefore many orders of magnitude larger than the incident drive bunch.

The results of the Trojan laser intensity scan are shown in figure 6 . In a) it is seen that the emittance increases slightly as the laser intensity increases from $a_{0}=0.016$ to $a_{0}=0.022$. Again, the observed values have been corrected by adding the laser emittance increase contribution, i.e. $\epsilon_{n} \approx 2.26 \times 10^{-8} \mathrm{mrad}$ for $a_{0}=0.016, \epsilon_{n} \approx 2.54 \times 10^{-8} \mathrm{mrad}$ for $a_{0}=0.018, \epsilon_{n} \approx 2.83 \times 10^{-8} \mathrm{mrad}$ for $a_{0}=0.020$, and $\epsilon_{n} \approx 3 \times 10^{-8} \mathrm{mrad}$ for $a_{0}=0.022$. However, this moderate increase of emittance is overcompensated by the increased and trapped HIT charge and currents, which amount to $Q \approx 2.3 \mathrm{pC}$ and $I \approx 2.5 \mathrm{kA}$ for $a_{0}=0.016, Q \approx 11 \mathrm{pC}$ and $I \approx 9.7 \mathrm{kA}$ for $a_{0}=0.018, Q \approx 40 \mathrm{pC}$ and $I \approx 27 \mathrm{kA}$ for $a_{0}=0.020$ and $Q \approx 110 \mathrm{pC}$ and $I \approx 73 \mathrm{kA}$ for $a_{0}=0.022$. In other words, the enhanced current wins over the increased emittance.

All these values for emittance and brightness are dramatically better than those known from typical plasma accelerators, but even from state-of-the-art conventional accelerators. While the confidence level is high from theory and simulations that such values of emittance and brightness can be produced via the Trojan Horse method $[19,20,23,26,28,29]$, a challenge will be to extract the bunches from the plasma, and to transport them towards the beamline downstream of the plasma, e.g. to the undulator.

\section{EXPERIMENTAL CHALLENGES AND OPPORTUNITIES OF TROJAN BUNCH GENERATION}

In order to realize Trojan Horse underdense photocathode electron bunches, the necessary ingredients are a) a dual or multi-component plasma medium, b) a high-current electron bunch and c) a synchronized release laser pulse.

A multi-component plasma is not a fundamental obstacle, because apart from hydrogen each chemical element has higher ionization thresholds that can be harvested to liberate additional electrons locally by the laser pulse. Also, 

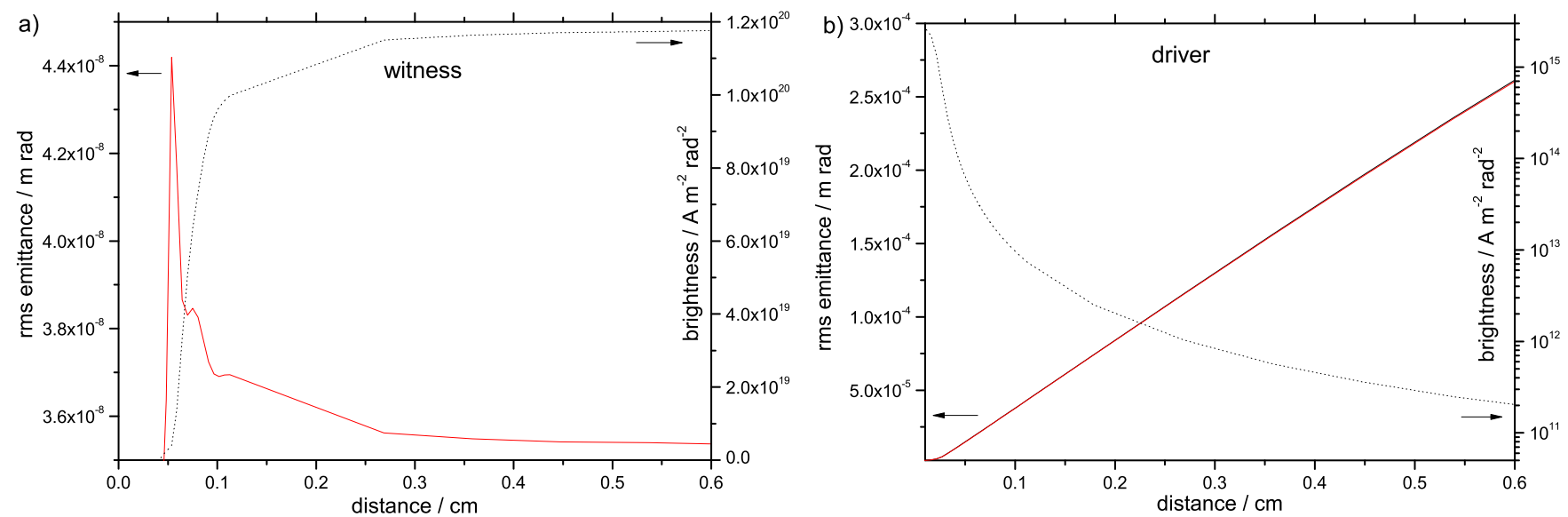

FIG. 5: Driver and witness emittance and brightness evolution during acceleration. a) Witness: after bunch generation, the emittance is decreased to values of $\epsilon_{n} \approx 3.6 \times 10^{-8} \mathrm{mrad}$, while the brightness rises to values of $B \approx 1.2 \times 10^{20} \mathrm{Am}^{-2} \mathrm{rad}^{-2}$, b) driver: the driver starts with an emittance of $\epsilon_{n}=2.25 \times 10^{-6} \mathrm{mrad}$, and during energy transfer to the witness the emittance is compromised due to transverse forces (compare figure $4 \mathrm{f}$ ), and the rising emittance leads to a strongly decreasing brightness (note the log scale for the brightness plot).
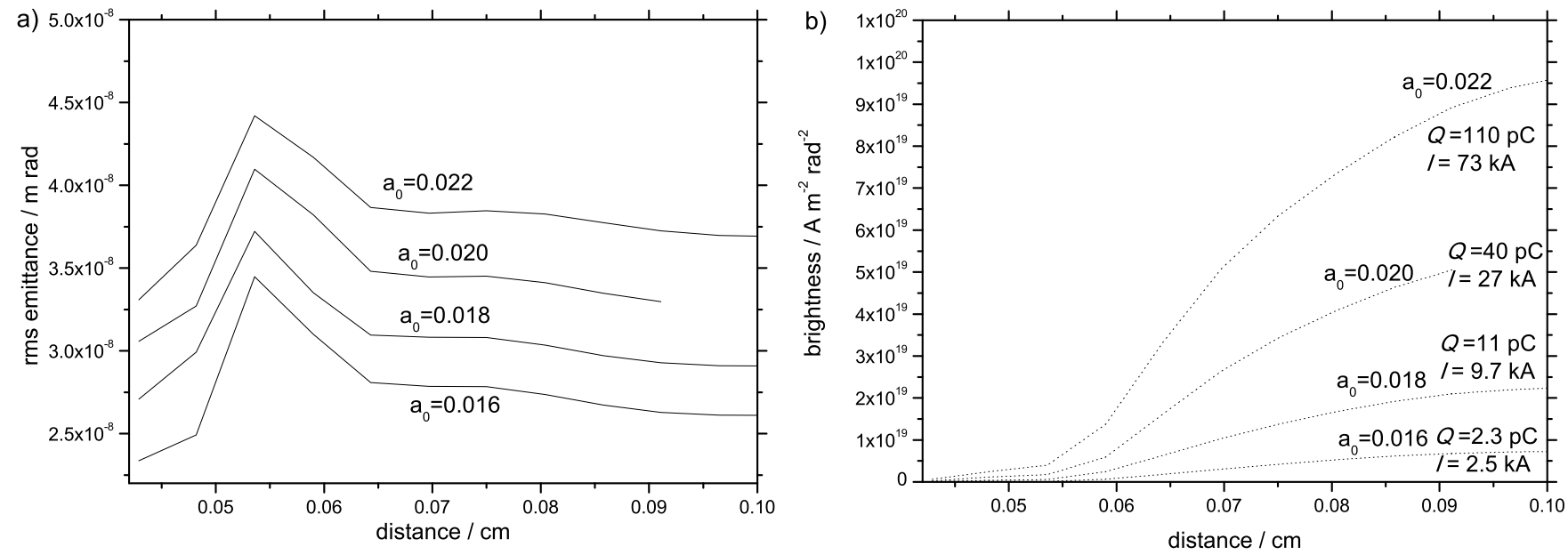

FIG. 6: The effect of laser intensity on emittance and brightness. a) the emittance increases as laser intensity increases from $a_{0}=0.016$ to $a_{0}=0.022, \mathrm{~b}$ ) nevertheless the brightness increases as the laser intensity increases due to a strongly increased current due to higher charge being released as laser intensity increases.

dual-component mixtures are today state-of-the-art in LWFA methods where HIT levels are ionized by the laser pulse intensity maximum or an additional high(er) intensity laser pulse [30-38]. In these laser-based methods injection and trapping of these electrons occurs because their release position may be located in the trapping potential region (or their trajectories can be slightly altered), which can be especially large with laser pulse drivers and their rather spherical bubbles. To the best of our knowledge, the use of a lower laser wavelength for the pump pulse and a shorter wavelength injection pulse is first mentioned in [30], and is today reconsidered [38].

The underdense photocathode provides a unique method to release electrons directly in the middle of the blowout at the maximized trapping potential, which optimally reduces the requirements on the drive bunch for trapping. Nevrtheless, providing electrons bunches with currents that can excite the plasma to produce wakefields with potentials exceeding the trapping potential in the first place is one of the biggest challenges even for the Trojan Horse method. A prime example of such a high current bunch is the bunch at FACET/SLAC, which is based on a thermionic cathode. Here, massive magnetic compression is the key to the short electron bunch durations and high currents needed for advanced PWFA. The introduction and establishment of photocathodes instead of thermal cathodes in 
linear accelerators allows for very high brightness, short pulses and repetition rates. Nearly all free-electron lasers (one remarkable exception is SACLA, which is based on a thermionic cathode) such as the LCLS and the upcoming XFEL and SwissFEL are based on such modern photoguns. An optimization focus in these systems is typically the emittance and energy spread next to the brightness, and these systems do often not operate above the current threshold for trapping in plasma waves. For PWFA, the optimization goals of electron bunches should be different from these systems which aim to provide FEL-capable electron bunches. The demands are peak current, with emittance and energy spread being of secondary concern. For example, energy spread is crucial in FEL systems because too large an energy spread prevents proper microbunching. In contrast, the energy spread practically does not matter for bunch drivers to be applied in the PWFA. The acceleration lengths are short, and the bunch electrons propagate with very similar $\beta$ despite huge energy differences. The bunch lengthening $\Delta L$ of an electron bunch with a certain energy spread $\Delta \gamma$ can be approximated as $\Delta L \approx L \times \Delta \gamma / \gamma^{3}$. It can be seen that at elevated energies of a few hundreds of $\mathrm{MeV}$ the lengthening amounts to less than a micron even over metre-scale acceleration distances and even at tens of percent energy spread. Therefore the drive beam peak current reduction due to energy spread is negligible in most cases. The same argument has been used in the above discussion that dephasing due to energy difference between driver and witness is not a practical issue in PWFA, in contrast to the LWFA (compare figure 2 b). Similarly, the emittance does not need to be optimized to values much better than $\epsilon_{n} \times 10^{-5}$ mrad for analogous reasons (compare figure 2 a). As a side note, both the emittance and the diffraction problem in the LWFA is even more critical at longer laser wavelengths due to the scaling of group velocity and Rayleigh length). The optimization of current instead of emittance and energy spread goes counter to the typical optimization goals of generations of electron linac systems and decade-long R\&D. It is hoped that this trade-off will allow in the future for the construction of electron guns that are specially designed to provide electron bunch drivers for PWFA applications. Increasing the charge released in a photocathode, e.g. via nano-structuring of the cathode material [39-41] which at the same time also would lead to higher space charge forces, may be one helpful ingredient to achieve higher peak currents.

Using such lower-quality electron bunch drivers for PWFA to allow for underdense photocathode Trojan Horse plasma wakefield acceleration is schematically shown in figure 7. The high current electron bunch driver, coming from conventional electron linacs with an energy of few tens of MeV up to tens of $\mathrm{GeV}$ (pink) drives a LIT plasma wave. A low-energy short pulse laser, synchronized to the electron bunch, provides the HIT electrons during the underdense photocathode process, producing the ultrahigh brightness electron bunch. The electron driver is then further accelerating the system, and in the optimized case, using enhanced transformer ratio and a high-charge, highenergy driver puse, the high brightness witness bunch will have energies up to $\sim 100 \mathrm{GeV}$. This high brightness beam is then sent to the undulator, where it may beget a 5th generation light source based on its high brightness and short pulses.

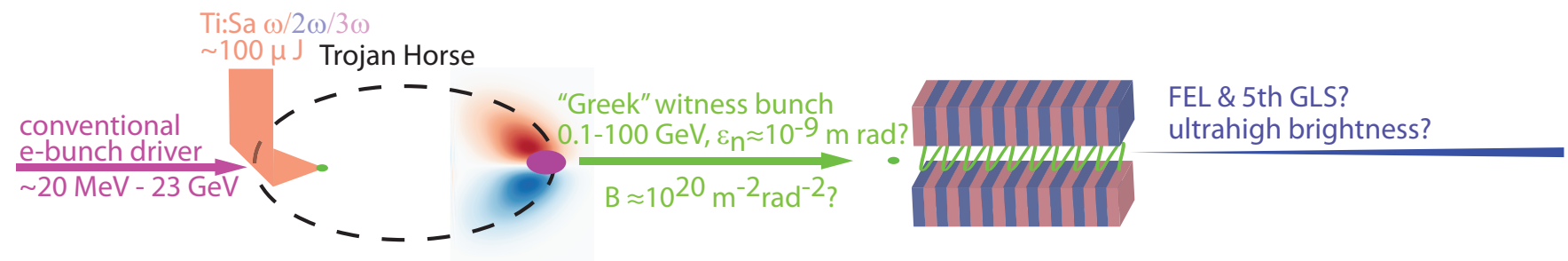

FIG. 7: Schematic setup for a Trojan Horse-driven undulator light source. A conventional electron bunch driver sets up the LIT plasma wave, and a low-energy, sub-relativistic intensity laser pulse releases the ultracold witness bunch

based on localized HIT component ionization directly within the LIT plasma wave. When trapped, the witness

bunch is accelerated to huge energies and brightness, which may then beget an ultrabright photon source when sent to appropriate undulators.

The requirements put on PWFA-capable electron bunches are fortunately highly complementary to the natural characteristics of the electron bunch output from typical LWFA systems. The orders of magnitude smaller size of plasma bubbles $(\sim 100 \mu \mathrm{m})$ when compared with rf-power metallic cavities (tens of cm) automatically leads to ultrashort, high-current electron bunches produced in LWFA. Currents up to $\sim 5 \mathrm{kA}$ have already been measured $[42,43]$. While the electron bunch output from LWFA can have in ideal cases energy spreads $<1 \%$ [42] and emittances down to $\epsilon_{n} \approx 10^{-7} \operatorname{mrad}[44]$, typically in practice one encounters energy spreads of a few to a few tens of percent, and emittances limited to the $\epsilon_{n} \approx w_{0} a_{0} / 2^{3 / 2} \approx 10^{-5}-10^{-6} \mathrm{mrad}$ range. This is because of the larger spot sizes of $w_{0} \gtrsim 10 \mu \mathrm{m}$ and intensities $a_{0} \gtrsim 1$, and because this laser interacts with the plasma electrons before they may be injected into the bubble via various schemes. However, as discussed above, a large energy spread and a limited emittance do not compromise the suitability of these bunches as drivers for PWFA. It has therefore been suggested as 
a general scheme to use LWFA-produced bunches as drivers for PWFA in hybrid LWFA $\rightarrow$ PWFA plasma accelerators [45]. This would conceptually allow for truly compact systems, whereas in hybrid linac $\rightarrow$ PWFA accelerators the compactness of the system is somewhat compromised because prior to the PWFA stage a conventional accelerator with a comparably large spatial footprint is needed.

Using LWFA to produce the electron bunch drivers for a subsequent Trojan Horse PWFA stage therefore seems like an attractive future option $[18,19]$. It would make the Trojan Horse method accessible for virtually every LWFA system worldwide, not only for the handful of PWFA-capable conventional linacs. Figure 8 shows the conceptual design of such a hybrid plasma accelerator based undulator light source. Current-optimized LWFA produces an electron bunch of moderate quality (e.g., large energy spread), which then acts as a driver for the underdense photocathode Trojan Horse stage. The laser pulse would be split off from the main LWFA drive laser and would therefore have inherently perfect synchronization with the electron bunch driver, because both, driver and witness, would then be produced by the same laser pulse. This is another fundamental advantage when compared to the linac $\rightarrow$ Trojan scheme, where the synchronization between electron bunch driver from the conventional linac and the underdense photocathode laser pulse is limited. Fortunately, laser-electron beam synchronization is a mainstream R\&D research direction at conventional accelerators, because there is a strong demand for ultra exact pump-probe systems for example from the FEL user perspective, and a synchronization of $<30 \mathrm{fs}$ or even better is believed to be feasible. The hybrid LWFA $\rightarrow$ Trojan scheme, however, would allow for synchronization $<1$ fs, depending partially on how well the LWFA process can be controlled. Such a system would in the final analysis be all-optical, using multiple laser beams: the main LWFA laser pulse, a preionization laser pulse for the PWFA stage, one or more [29] underdense photocathode lasers, probe lasers to monitor the interaction and/or for pump/probing of the sample behind the undulator, and optionally for seeding the FEL process in the undulator (e.g. via high harmonic generation). It shall be noted here that the strictly collinear scheme may lead to some complications because of diffractive effects [46] in case of the short bunches obtainable from LWFA systems and the correspondingly short matched plasma wavelengths, albeit this would not be a showstopper and could be overcome for exmple by using a slight angle between underdense photocathode laser and beam propagation axis [18].

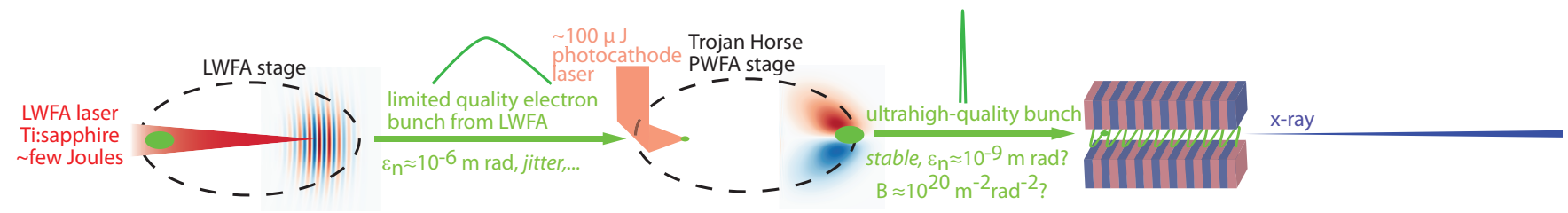

FIG. 8: Schematic setup for a Trojan Horse stage, driven by an electron beam from a laser-plasma-accelerator.

Now, while the compactness, the inherently perfect synchronization, and ubiquity of laser-plasma-accelerators are strong advantages of developing hybrid LWFA $\rightarrow$ Trojan systems, there are currently two major disadvantages. One is the low repetition rate of today's high-power laser systems, which are typically $10 \mathrm{~Hz}$, in comparison to the hundreds of $\mathrm{Hz}$ or even many $\mathrm{kHz}$ conventional systems. The other is the stability and controllability of the LWFA process. However, the latter issue has been continuously improved by the community [47-49], and there is confidence that progress will continue. As regards the repetition rate, higher repetition rate laser systems do already appear on the fringes of the technological horizon. On the one hand, these are OPCPA-type laser systems with repetition rates up to the $\mathrm{kHz}$ range, on the other hand there are thin-disk and fibre lasers [50, 51]. Coherent [51] or incoherent [52] combination of multiple fibre lasers and/or renewed interest in multi-pulse schemes [53] may in the future lead to the realization of efficient high repetition rate LWFA systems. While it is unclear whether such systems would allow at the same time for enhanced electron beam quality in direct LWFA schemes, they may nevertheless be good sources for hybrid LWFA $\rightarrow$ Trojan systems, based on their relaxed beam quality requirements.

The above discussion is concentrated on the tunability, ultrashort duration and ultrahigh brightness of obtainable electron bunches. While increased electron brightness is clearly a highly attractive feature for future light sources, and may in principle at elevated energies $\gamma$ allow lasing at wavelengths down to $\lambda_{\min } \approx 4 \pi \epsilon_{n} \approx 0.1 \AA$ or below [19]. However, energy spread has not been discussed, which can be a show-stopper for FEL processes. As indicated in [19], beam loading is an attractive way to reduce the energy spread. While also being a prominent effect for laserplasma-accelerators, dephasing makes it difficult to exploit beam loading properly. In PWFA systems, where in the absence of significant dephasing one has a quasistatic system, beam loading is much more straightforward. In fact, in recent research [29] it has been shown that multiple electron bunches can be generated with multiple underdense photocathode laser pulses. This allows not only for a tunable and flexible multibunch train generation, but also to design the shape an electron bunch witness (train) current profile which is well-suited to provide for beam loading. This said, the limits of the energy spread obtainable with this method are not well known, and will be explored in 
ongoing and future R\&D.

An alternative light source may be hybrid systems with inverse Compton scattering (ICS), where the ultrashort, high brightness electron pulses obtainable from the Trojan would be exploited by scattering with a high-power laser pulse as shown in figure 9 .

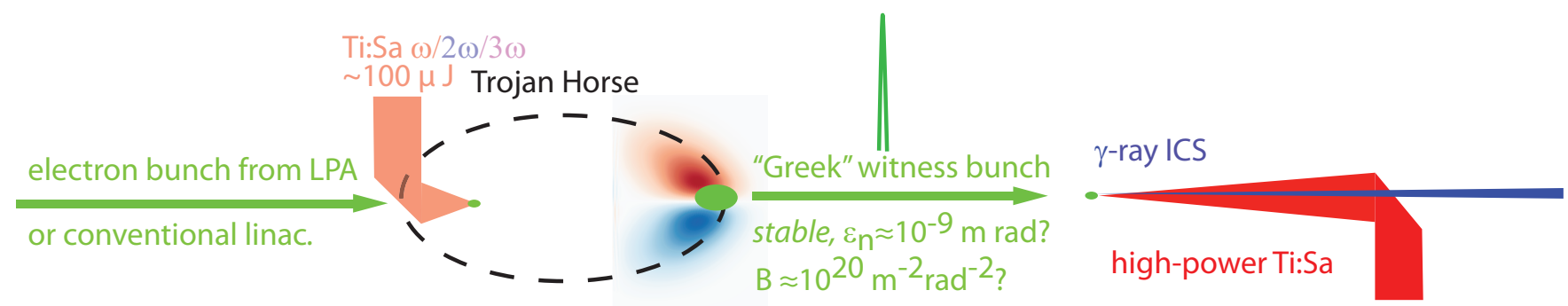

FIG. 9: Schematic setup for an inverse Compton scattering system.

Such a scheme would profit from inherent synchronization in case of an LWFA $\rightarrow$ Trojan hybrid, and would be more forgiving as regards energy spread of the produced electron bunches when compared to FEL processes. Furthermore in contrast to FEL light sources, where the electron beam has to be extracted from the plasma and transported to the undulator with the challenges of emittance and general beam quality preservation, the inverse Compton scattering light source can happen in the plasma itself. This would be compatible with the enormous currents (compare figure 6 ) and transverse bunch sizes, which may only be supported inside the plasma.

\section{SUMMARY AND OUTLOOK}

Differences of LWFA and PWFA, and the advantages of using laser pulses and electron bunches, respectively, for plasma wakefield acceleration are discussed. In the context of the "Trojan Horse" underdense photocathode plasma wakefield acceleration, the advantages are combined in such a way that the long, dephasing-free acceleration lengths obtainable from high-current electron drive bunches set up the plasma blowout cavity, and that the short Rayleigh lengths of focused laser pulse are used to release ultracold photoelectrons inside the blowout. The electron bunch driver can come from conventional linacs, or from laser-plasma-accelerators. Bunches from conventional linacs have the advantage of stablity and high repetition rate, but the challenge of synchronization between the electron bunch, the rf phase, and the underdense photocathode laser pulse, and the challenge of producing sufficient current to trap Trojan electrons. Especially at lower electron energies, which may be attractive because then the linac can be shorter and the total footprint of the hybrid system is smaller, space charge effects may limit the obtainable peak current. It is proposed to explore whether a "PWFA-mode" of linacs can be developed, where the design goals would be on (temporary) peak current rather than on emittance and energy spread of the drive bunches, which play a secondary role in PWFA. Electron bunches from laser-plasma-accelerators typically have rather large energy spread and emittance, but also very high currents. Using these bunches as drivers for PWFA in general, and for Trojan Horse in particular, has the advantage of truly compact systems, and of inherently perfect synchronization between the electron bunch driver and the photocathode laser pulse. Drawbacks are the limited stability and repetition rate from today's LWFA systems. Remedy may come from future high repetition rate laser systems.

The enormous electron brightness obtainable from the Trojan exceeds state-of-the-art accelerators by orders of magnitude. It is therefore highly promising for next generation light sources, for example free-electron lasers or inverse Compton scattering schemes. While the latter can operate also with larger energy spreads, free-electron lasing may not work with too large energy spreads, or work at compromised performance. The high tunability of underdense photocathodes, however, together with the use of multiple laser pulses, can design electron bunches with matched beam loading, which allows to reduce the energy spread. Therefore conceptually, the Trojan Horse may be able to challenge the three main problems of plasma accelerators today: stability\&tunability, brightness and energy spread. Optimized "designer" electron bunches may then be used as drivers for 5th generation light sources in the future. Collaborative efforts are underway to demonstrate the Trojan Horse at FACET/SLAC, and to implement it other conventional systems which plan to engage in PWFA in the future, such at DESY, Daresbury Laboratory and INFN Frascati. In parallel, the potential refinement of laser-plasma-accelerators to hybrid Trojan Horse accelerators and light sources is explored. 


\section{ACKNOWLEDGMENTS}

This work was supported by DFG, by EPSRC EP/J018171/1, STFC No. 4070022104, DOE DE-SC0009533, DEFG02-07ER46272 DE-FG03-92ER40693, by ONR Contract No. N00014-06-1-0925 and Helmholtz VH-VI-503. We acknowledge the assistance of the VORPAL development team. This research used computational resources of the National Energy Research Scientific Computing Center, which is supported by DOE DE-AC02- 05CH11231, and of JUROPA, and of HLRN. D.A.J. acknowledges the support of the U.K. EPSRC (EP/J018171/1) and the EC's 7th Framework Programme (LASERLAB-EUROPE no. 284464, the EUCARD-2 project (no. 312453) and the Extreme Light Infrastructure (ELI) project).

[1] W. C. Rntgen, Sonderabdruck aus den Sitzungsberichten der Wrzburger Physikalisch-Medizinischen Gesellschaft (1896).

[2] I. Langmuir, Proc. Natl. Acad. USA 14, 627 (1928).

[3] L. Tonks and I. Langmuir, Phys. Rev. 33, 195 (1929), URL http://link.aps.org/doi/10.1103/PhysRev.33.195.

[4] L. Tonks, American Journal of Physics 35, 857 (1967), URL http://link.aip.org/link/?AJP/35/857/1.

[5] V. Veksler (AIP, Geneva, 1956), vol. 1, p. 80.

[6] T. Tajima and J. M. Dawson, Phys. Rev. Letters 43, 267 (1979).

[7] P. Chen, J. M. Dawson, R. W. Huff, and T. Katsouleas, Phys. Rev. Lett. 54, 693 (1985), URL http://link.aps.org/ doi/10.1103/PhysRevLett.54.693.

[8] J. B. Rosenzweig, D. B. Cline, B. Cole, H. Figueroa, W. Gai, R. Konecny, J. Norem, P. Schoessow, and J. Simpson, Phys. Rev. Lett. 61, 98 (1988), URL http://link.aps.org/doi/10.1103/PhysRevLett.61.98.

[9] A. Pukhov and J. Meyer-ter Vehn, Applied Physics B-Lasers and Optics 74, 355 (2002).

[10] H. A. H. Boot and R. B. R.S.-Harvie, Nature 180, 1187 (1957).

[11] N. Iwata and Y. Kishimoto, Phys. Rev. Lett. 112, 035002 (2014), URL http://link.aps.org/doi/10.1103/PhysRevLett. 112.035002.

[12] E. Esarey, C. B. Schroeder, and W. P. Leemans, Rev. Mod. Phys. 81, 1229 (2009), URL http://link.aps.org/doi/10. 1103/RevModPhys.81.1229.

[13] D. Strickland and G. Mourou, Optics Communications 56, 219 (1985).

[14] W. P. Leemans et al., Nature Physics 2, 696 (2006).

[15] S. Karsch, J. Osterhoff, A. Popp, T. P. Rowlands-Rees, Z. Major, M. Fuchs, B. Marx, R. Horlein, K. Schmid, L. Veisz, et al., New Journal Of Physics 9, 415 (2007).

[16] X. Wang, R. Zgadzaj, N. Fazel, Z. Li, S. A. Yi, X. Zhang, W. Henderson, Y.-Y. Chang, R. Korzekwa, H.-E. Tsai, et al., Nat Commun 4, (2013).

[17] I. Blumenfeld et al., Nature 445, 741 (2007).

[18] B. Hidding, G. Pretzler, D. Bruhwiler, and J. Rosenzweig, Method for generating electron beams in a hybrid plasma accelerator (2011), german Patent DE 102011104 858.1, US/PCT patent Ser. No. PCT/US12/043002.

[19] B. Hidding, G. Pretzler, J. B. Rosenzweig, T. Königstein, D. Schiller, and D. L. Bruhwiler, Phys. Rev. Lett. 108, 035001 (2012), URL http://link.aps.org/doi/10.1103/PhysRevLett.108.035001.

[20] B. Hidding, J. B. Rosenzweig, Y. Xi, B. O'Shea, G. Andonian, D. Schiller, S. Barber, O. Williams, G. Pretzler, T. Königstein, et al., AIP Conference Proceedings 1507, 570 (2012), URL http://link.aip.org/link/?APC/1507/570/1.

[21] T. Katsouleas, Phys. Rev. A 33, 2056 (1986).

[22] A. Caldwell et al., Nature Physics 5, 363 (2009).

[23] Y. Xi, B. Hidding, D. Bruhwiler, G. Pretzler, and J. B. Rosenzweig, Phys. Rev. ST Accel. Beams 16, 031303 (2013), URL http://link.aps.org/doi/10.1103/PhysRevSTAB.16.031303.

[24] T. Katsouleas, S. Wilks, P. Chen, J. Dawson, and J. Su, Particle Accelerators 22, 81 (1987).

[25] M. Tzoufras, W. Lu, F. S. Tsung, C. Huang, W. B. Mori, T. Katsouleas, J. Vieira, R. A. Fonseca, and L. O. Silva, Phys. Rev. Lett. 101, 145002 (2008), URL http://link.aps.org/doi/10.1103/PhysRevLett.101.145002.

[26] X. L. Xu et al., Phys. Rev. Lett. 112, 035003 (2014), URL http://link.aps.org/doi/10.1103/PhysRevLett.112.035003.

[27] C. Nieter and J. R. Cary, Journal of Computational Physics 196, 448 (2004), ISSN 0021-9991, URL http://www. sciencedirect.com/science/article/B6WHY-4B954G3-1/2/675d7d4de2f99f1448034cb90ce46f8e.

[28] F. Li, J. F. Hua, X. L. Xu, C. J. Zhang, L. X. Yan, Y. C. Du, W. H. Huang, H. B. Chen, C. X. Tang, W. Lu, et al., Phys. Rev. Lett. 111, 015003 (2013), URL http://link.aps.org/doi/10.1103/PhysRevLett.111.015003.

[29] B. Hidding et al., arXiv p. 1403.1109 (2014).

[30] D. Umstadter, J.-K. Kim, and E. Dodd, Method and apparatus for generating and accelerating ultrashort electron pulses (1995), uS patent Ser. No. 5,789,876.

[31] M. Chen, Z.-M. Sheng, Y.-Y. Ma, and J. Zhang, Journal of Applied Physics 99, 056109 (2006), ISSN 00218979, URL http://dx.doi.org/10.1063/1.2179194.

[32] D. Umstadter, J. K. Kim, and E. Dodd, Phys. Rev. Lett. 76, 2073 (1996), URL http://link.aps.org/doi/10.1103/ PhysRevLett.76.2073. 
[33] C. McGuffey, A. G. R. Thomas, W. Schumaker, T. Matsuoka, V. Chvykov, F. J. Dollar, G. Kalintchenko, V. Yanovsky, A. Maksimchuk, K. Krushelnick, et al., Phys. Rev. Lett. 104, 025004 (2010).

[34] A. Pak, K. A. Marsh, S. F. Martins, W. Lu, W. B. Mori, and C. Joshi, Phys. Rev. Lett. 104, 025003 (2010), URL http://link.aps.org/doi/10.1103/PhysRevLett.104.025003.

[35] C. E. Clayton, J. E. Ralph, F. Albert, R. A. Fonseca, S. H. Glenzer, C. Joshi, W. Lu, K. A. Marsh, S. F. Martins, W. B. Mori, et al., Phys. Rev. Lett. 105, 105003 (2010).

[36] M. Chen, E. Esarey, C. B. Schroeder, C. G. R. Geddes, and W. P. Leemans, Physics of Plasmas (1994-present) 19, 033101 (2012).

[37] N. Bourgeois, J. Cowley, and S. M. Hooker, Phys. Rev. Lett. 111, 155004 (2013), URL http://link.aps.org/doi/10. 1103/PhysRevLett.111.155004.

[38] L.-L. Yu, E. Esarey, C. Schroeder, J.-L. Vay, C. Benedetti, C. Geddes, M. Chen, and W. Leemans, Phys. Rev. Lett. 112, 125001 (2014), URL http://link.aps.org/doi/10.1103/PhysRevLett.112.125001.

[39] R. K. Li, H. To, G. Andonian, J. Feng, A. Polyakov, C. M. Scoby, K. Thompson, W. Wan, H. A. Padmore, and P. Musumeci, Phys. Rev. Lett. 110, 074801 (2013), URL http://link.aps.org/doi/10.1103/PhysRevLett.110.074801.

[40] A. Polyakov, C. Senft, K. F. Thompson, J. Feng, S. Cabrini, P. J. Schuck, H. A. Padmore, S. J. Peppernick, and W. P. Hess, Phys. Rev. Lett. 110, 076802 (2013), URL http://link.aps.org/doi/10.1103/PhysRevLett.110.076802.

[41] G. Tang, A. C. Hourd, and A. Abdolvand, Applied Physics Letters 101, 231902 (2012), URL http://scitation.aip. org/content/aip/journal/apl/101/23/10.1063/1.4769215.

[42] S. M. Wiggins, R. C. Issac, G. H. Welsh, E. Brunetti, R. P. Shanks, M. P. Anania, S. Cipiccia, G. G. Manahan, C. Aniculaesei, B. Ersfeld, et al., Plasma Physics and Controlled Fusion 52, 124032 (2010), URL http://www.ingentaconnect. com/content/iop/ppcf/2010/00000052/00000012/art124032.

[43] O. Lundh, J. Lim, C. Rechatin, L. Ammoura, A. Ben-Ismail, X. Davoine, G. Gallot, J.-P. Goddet, E. Lefebvre, V. Malka, et al., Nat Phys 7, 219 (2011), URL http://dx.doi.org/10.1038/nphys1872.

[44] G. R. Plateau, C. G. R. Geddes, D. B. Thorn, M. Chen, C. Benedetti, E. Esarey, A. J. Gonsalves, N. H. Matlis, K. Nakamura, C. B. Schroeder, et al., Phys. Rev. Lett. 109, 064802 (2012), URL http://link.aps.org/doi/10.1103/ PhysRevLett.109.064802.

[45] B. Hidding, T. Königstein, J. Osterholz, S. Karsch, O. Willi, and G. Pretzler, Phys. Rev. Lett. 104, 195002 (2010).

[46] A. G. R. Thomas, C. D. Murphy, S. P. D. Mangles, A. E. Dangor, P. Foster, J. G. Gallacher, D. A. Jaroszynski, C. Kamperidis, K. L. Lancaster, P. A. Norreys, et al., Phys. Rev. Lett. 100, 255002 (2008), URL http://link.aps.org/doi/10. 1103/PhysRevLett.100.255002.

[47] J. Osterhoff, A. Popp, Z. Major, B. Marx, T. P. Rowlands-Rees, M. Fuchs, M. Geissler, R. Hrlein, L. Veisz, B. Hidding, et al., accepted for publication in Physical Review Letters (2008).

[48] W. Yan, L. Chen, D. Li, L. Zhang, N. A. M. Hafz, J. Dunn, Y. Ma, K. Huang, L. Su, M. Chen, et al., Proceedings of the National Academy of Sciences 111, 5825 (2014), http://www.pnas.org/content/111/16/5825.full.pdf+html, URL http://www.pnas.org/content/111/16/5825. abstract.

[49] S. Banerjee, S. Y. Kalmykov, N. D. Powers, G. Golovin, V. Ramanathan, N. J. Cunningham, K. J. Brown, S. Chen, I. Ghebregziabher, B. A. Shadwick, et al., Phys. Rev. ST Accel. Beams 16, 031302 (2013), URL http://link.aps.org/ doi/10.1103/PhysRevSTAB.16.031302.

[50] C. Jaurgeui, J. Limpert, and A. Tuennermann, Nature Photonics 7, 861 (2013).

[51] G. Mourou, B. Brocklesby, T. Tajima, and J. Limpert, Nature Photonics 7, 258 (2013).

[52] C. Benedetti, C. B. Schroeder, E. Esarey, and W. P. Leemans, Physics of Plasmas (1994-present) 21, 056706 (2014), URL http://scitation.aip.org/content/aip/journal/pop/21/5/10.1063/1.4878620.

[53] S. Hooker et al., arXiv p. 1401.7874 (2014). 